\title{
Tsafon
}

Revue d'études juives du Nord

$72 \mid 2016$

Juifs, Israéliens, dans la littérature française et israélienne

\section{Willy Bok, tel que je l'ai connu}

\section{Carol lancu}

\section{(2) OpenEdition}

Journals

Édition électronique

URL : https://journals.openedition.org/tsafon/372

DOI : $10.4000 /$ tsafon.372

ISSN : 2609-6420

Éditeur

Association Jean-Marie Delmaire

Édition imprimée

Date de publication : 1 décembre 2016

Pagination : 139-148

ISSN : 1149-6630

\section{Référence électronique}

Carol lancu, « Willy Bok, tel que je l'ai connu », Tsafon [En ligne], 72 | 2016, mis en ligne le 31 mai 2018, consulté le 29 juin 2021. URL : http://journals.openedition.org/tsafon/372 ; DOI : https://doi.org/ $10.4000 /$ tsafon. 372 


\section{Hommage}

\section{Willy Bok, tel que je l'ai connu}

\section{Carol Iancu*}

J'ai fait la connaissance de Willy Bok à Paris, en 1979, et lors de cette première rencontre notre entretien concerna l'histoire des Juifs de l'Europe de l'Est. Dès le 24 juillet de la même année, je lui transmettais une liste d'articles et d'ouvrages sur les Juifs de Pologne susceptibles d'être utiles pour une bibliographie qu'il était en train de préparer. En même temps, faisant suite à sa demande, je lui proposais quelques sujets pour des conférences à donner dans le cadre de l'Institut Universitaire d'Études du Judaïsme Martin Buber, à Bruxelles. Par courrier séparé, je lui faisais parvenir mon premier livre sur les Juifs de Roumanie ${ }^{1}$ et plusieurs tirés-à-part de mes études parues dans la Revue d'Histoire diplomatique $^{2}$, Toladot ${ }^{3}$ et la Revue des études juives ${ }^{4}$. Le 27 août

\footnotetext{
* Université Paul Valéry - Montpellier 3, directeur de l'École des Hautes Études du Judaïsme, professeur associé à l'Université de Bucarest.

Willy J.-L. Bok, fut directeur de l'Institut d'Études du Judaïsme (Institut Martin Buber) de Bruxelles de 1972 à 2002, il était né à Liège en 1935, il est décédé à Bruxelles le 5 novembre 2015. En guise d'hommage, Carol Iancu nous propose un article témoignage intitulé « Willy Bok, tel que je l'ai connu ». (Note de la rédaction).

${ }^{1}$ Les Juifs en Roumanie (1866-1919). De l'exclusion l'émancipation, Aix-en-Provence, Éditions de l'Université de Provence, 1979, 382 p. Préface de Pierre Guiral.

2 « Napoléon III et la politique française à l'égard de la Roumanie », Revue d'Histoire diplomatique, 1974, n¹-2, pp. 59-85.

3 «L'intérêt pour la colonisation d'Eretz Israël dans la lettre d'un médecin juif de Giurgiu adressée à la société l'Alliance israélite universelle de Paris (1867) », Toladot, Jérusalem, 1976, n¹2-13, pp. 38-42 (en roumain) et pp. 4-8 (en hébreu).

4 «Adolphe Crémieux, l'Alliance israélite universelle et les Juifs de Roumanie au début $\mathrm{du}$ règne de Carol Hohenzollern Sigmaringen », Revue des études juives, 1974, t. CXXXIII (3-4), pp. 481-502 ; «Une lettre inédite d'Éléazar Rokéah sur les débuts du mouvement Ychouv Eretz Israel (1880)», Revue des études juives, 1976, t. CXXXV(1-
} 
toujours de la même année, il répondait à ma lettre en me proposant de venir au mois de décembre pour traiter du thème: «Les Juifs en Roumanie, de l'exclusion à l'émancipation ». Ce fut le début d'une vaste correspondance qui s'est poursuivie pendant trente-cinq ans et que j'espère faire publier dans un avenir pas très lointain. Cette correspondance est le témoignage privilégié non seulement de l'amitié forte qui s'est nouée entre nous, mais aussi et surtout de ses multiples activités, des thèmes de recherches sur lesquels il travaillait, de la diversité et de la richesse des enseignements dispensés à l'Institut qu'il dirigeait avec rigueur et une courtoisie et une gentillesse qui ont suscité l'admiration de tous les enseignants et tous les étudiants. C'est à travers cette correspondance que je souhaite faire ressortir sa personnalité.

Mon premier cours sur les Juifs de Roumanie fut annoncé dans les Nouvelles de l'Institut Martin Buber, et sa teneur fit l'objet d'une publication officielle sous forme d'une brochure ${ }^{5}$. Très intéressé par l'histoire des Juifs de mon pays d'origine qui représenta longtemps une tache blanche dans l'historiographie du judaïsme européen, il m'encouragea dans mes projets de publication. Dans une lettre du 20 décembre 1979, il m'assurait de son soutien, notamment pour la publication d'une correspondance entre Adolphe-Isaac Crémieux et Gerson von Bleichröder, consacrée au combat pour l'émancipation des Juifs de Roumanie à l'occasion du Congrès de Berlin, que j'avais découverte dans les archives de l'Alliance israélite universelle de Paris. Cet ouvrage devait paraître plus tard aux éditions de l'université Paul Valéry de Montpellier6.

Plusieurs personnes d'origine roumaine ont assisté à mes premières conférences et, grâce à Willy Bok, un « Comité belge pour l'histoire des Juifs de Roumanie » a pu se constituer et se réunir dans une salle de 1'Institut Buber. Parmi les membres de ce Comité qui s'est rassemblé une première fois le 8 décembre 1981, il convient de citer Joseph Halévy, fils de l'ancien grand rabbin Meyer Abraham Halévy, et Messieurs Aristide Steinhart, Marcel Kirschen, Josif Herscovici, Emmanuel Neuman, Carol Stelian, L.M. Schrager, Radu Apostol et Andrei Laude. Ce petit Comité

3), pp. 177-183 ; «Le Serment More judaïco en Roumanie », Revue des études juives, 1976, t. CXXXV (1-3), pp. 169-176.

5 Les Juifs en Roumanie. De l'exclusion à l'émancipation, Bruxelles, éditions de l'Institut Martin Buber, 1980.

${ }^{6}$ Bleichröder et Crémieux. Le combat pour l'émancipation des Juifs de Roumanie devant le Congrès de Berlin. Correspondance inédite (1878-1880), Montpellier, université Paul Valéry, 1987, 264 p. (Sem n³). 
m'a donné l'idée de créer une "Société d'Études sur l'histoire des Juifs de Roumanie » fondée à Paris en 1986, avec plusieurs amis, parmi lesquels le regretté docteur Lazar Moscovici, survivant d'Auschwitz où il a soigné le poète Benjamin Fondane avant que celui-ci ne soit gazé le 2 octobre 1944, l'écrivain Edgar Reichman, le peintre Tudor Banus et Marie Bonnet. Willy Bok a soutenu cette initiative et a fait le lien entre le Comité belge et l'Association parisienne. Je possède une carte de visite de l'Institut Martin Buber avec cette annonce : «Réunion de la Société d'Histoire des Juifs de Roumanie : $3^{\text {ème }}$ étage, bureau $007 »$. Cette réunion eut lieu le 12 mai 1987, le jour même, avant ma conférence sur «L'émancipation des Juifs de Roumanie ». Aussitôt après (le 30 juillet), il m'écrivait : " Je crois aussi que progressivement la section belge de la Société pour l'histoire des Juifs de Roumanie se développera. Il serait peut-être souhaitable qu'un compte rendu de la réunion constitutive soit rédigé... ». Une année plus tard, nous avons pu organiser dans la capitale française, le 10 janvier 1988, une table ronde intitulée: «La Mémoire des Juifs de Roumanie ».

En marge de notre correspondance nous avons échangé nos publications. Le 11 octobre 1982, je remerciais Willy Bok pour le beau catalogue Charlotte Salomon. Leben oder Theater, contenant son étude approfondie «L'Allemagne et les Juifs (1919-1939)». En 1982, je l'invitais à Montpellier pour participer au colloque « Armand Lunel et les Juifs du Midi ». S'excusant de n'avoir pas pu y participer, il m'écrivait le 18 décembre 1982 :

Veuillez m'excuser de vous écrire avec un si grand retard. Je n'arrive jamais à
réaliser tout ce que je souhaite entreprendre, mais j'ai quelques circonstances
atténuantes pour me faire pardonner. Vous n'ignorez pas que l'Institut Martin
Buber est mon vampire « favori » et quotidien. En outre, j'ai été invité par L.
Poliakov et M. Ollender à faire un exposé sur l'antisémitisme aux États-Unis.
Cela m'a ouvert, hélas, de nouvelles perspectives de recherches et beaucoup de
travail. J'espère que je pourrai rédiger un article sur ce sujet dans les semaines à
venir. J'attends avec impatience votre deuxième volume sur l'histoire des Juifs
en Roumanie, car de plus en plus je suis convaincu de la nécessité d'étudier
l'antisémitisme nazi à la lumière des questions politiques de l'Europe centrale et
orientale. Je vous félicite pour l'initiative que vous avez prise de créer à
l'université de Montpellier le Certificat de Civilisation et Langue d'Israël. J'ai lu
attentivement le programme que vous m'avez envoyé et je le trouve excellent
(Merci d'avoir inclus mon article sur le Bouc'). L'ULB aussi a accepté d'inscrire

${ }^{7}$ Cf. Willy Bok, «Le bouc et le nomade. Essai sur le symbolisme du bouc dans la Bible », dans Les Mélanges Armand Abel, Leiden, Éditions E.J. Brill, 1978, pp. 1-15. 
à son programme de cours l'enseignement de l'hébreu contemporain, donné par Elyahou Reichert, et celui de l'histoire contemporaine que je consacre au judaïsme américain cette année...

En 1983, grâce à Willy Bok, je fis la connaissance de Maurice Konopnicki qui malheureusement nous a quitté le 23 juillet 2015, et qui devint aussi un ami, et plus tard de Georges Schnek (décédé le 18 mars 2012), le président du Consistoire central israélite de Belgique, avec lesquels j'ai échangé toute une correspondance.

En novembre 1984, Willy Bok m'envoyait sa belle étude sur «Chaïm Perelman ou la double fidélité ${ }^{8}$ et je lui faisais parvenir en retour mon étude sur " La communauté juive à Marseille », parue dans la revue Religion et Société ( $\left.{ }^{\circ} 20,1984\right)$. Le 28 décembre 1984, il me remerciait et m'annonçait l'envoi du recueil Dix années d'activités de l'Institut Martin Buber.

Le 18 mars 1985, je l'invitais au colloque international « Les Juifs à Montpellier et dans le Languedoc à travers l'histoire », prévu les 22 et 23 octobre 1985, à l'occasion du millénaire de la ville. Il me répondit le 20 juin 1985, soulignant sa surcharge de travail, je cite :

J'ai dû présenter récemment deux communications : l'une sur «Entrelacs de la religion et de la laïcité dans les milieux juifs en Belgique »" et l'autre sur «Orthopraxie et laïcité en Israël $»^{10}$. J'ai dû achever mon enseignement à l'ULB et faire passer des examens. Je dois avant la fin du mois terminer la rédaction de la $2^{\mathrm{e}}$ communication et revoir mon étude sur « La réhabilitation de l'adolescence dans la société juive». Avant la fin de l'été, je devrais pour bien faire avoir terminé l'étude biographique de Max Gottschalk que j'ai promise depuis longtemps...

Il put cependant venir pour présider une séance et eut la joie de recevoir la «Médaille du Millénaire» des mains du député-maire Georges Frêche. La première page des Nouvelles de l'Institut Martin Buber ( ${ }^{\circ}$ 90, novembre 1985), sous le titre «Le Millénaire de Montpellier et les études juives » rend compte de cet événement :

\footnotetext{
${ }^{8}$ Willy Bok, «Chaïm Perelman ou la double fidélité », La Pensée et les hommes, revue mensuelle de philosophie et de morale laïques, 1984-1985, $28^{\mathrm{e}}$ année, pp. 71-76.

${ }^{9}$ Paru sous le titre «Entrelacs du religieux et du laïc dans les milieux juifs en Belgique », dans La Belgique et ses Dieux. Églises, Mouvements religieux et laïques, Louvain-la-Neuve, Éditions Cabay, 1985, pp. 333-346.

10 Paru sous le titre «Orthodoxie, hétérodoxie et laïcité en Israël», dans Les Intégrismes, Bruxelles, 1986, Éditions de l'Université de Bruxelles, collection « La Pensée et les Hommes », pp. 87-110.
} 
La distinction offerte au directeur de l'Institut Martin Buber honore l'Institut tout entier. Elle est un témoignage de l'esprit de collaboration fructueuse qui préside aux relations établies entre l'Institut et le Centre de recherche et d'enseignement [du judaïsme] de l'Université Paul Valéry [de Montpellier].

Nos relations universitaires et personnelles se sont renforcées et il est venu à Montpellier pour assister à la Bar Mitsva de mon fils Michaël en avril 1986 ; quelque mois plus tard (le 22 septembre) il m'annonçait le décès de sa chère maman. La même année, il m'informait que l'Institut s'installait dans de nouveaux locaux, son Bulletin mensuel arrivait au $\mathrm{n}^{\circ} 100$, tandis que l'ULB agréait la création d'une « Licence en Histoire, pensée et civilisation juives ». En 1988, Willy Bok participait au Colloque international: Permanences et mutations dans la société israélienne, organisé à Montpellier à l'occasion du quarantième anniversaire de l'État d'Israël, avec une belle communication intitulée : «Le judaïsme libéral américain et Israël ${ }^{11}$. Le Bulletin de l'Institut Martin Buber du mois de mai de la même année présenta un bref mais élogieux compte rendu consacré à cette rencontre universitaire. Le 9 septembre 1988, il évoquait, dans une lettre, son voyage en Israël où il avait participé à un séminaire consacré à l'enseignement de la Shoah, organisé par le «Centre international pour l'enseignement de la civilisation juive dans les universités », dirigé par le professeur Moshe Davis.

À cette occasion il put revoir les professeurs Roberto Bachi, Usiel Oscar Schmeltz et Sergio Della Pergola. Il était très attaché à ces professeurs, avec lesquels il avait participé à deux colloques importants sur: La vie juive dans l'Europe contemporaine qui eurent lieu à Bruxelles en 1962 et en 1967. Lors du premier colloque, Willy Bok, alors chargé de recherches au Centre National des Hautes Études Juives de Bruxelles, y présenta une communication sur «Considérations sur les estimations quantitatives de la population juive en Belgique $»^{12}$. Au

\footnotetext{
${ }^{11}$ Cf. Willy Bok, « Le judaïsme libéral américain et Israël », dans Permanences et mutations dans la société israélienne, sous la direction de Carol Iancu, Montpellier, Centre de Recherches et d'Études juives et hébraïques, Université Paul Valéry, 1996, pp. 119-128.

Cf. Willy Bok «Considérations sur les estimations quantitatives de la population juive en Belgique ", dans La vie juive dans l'Europe contemporaine / Jewish Life in Contemporary Europe, Actes du Colloque tenu à l'Institut de Sociologie de l'Université libre de Bruxelles du 19 au 21 septembre 1962, Éditions de l'Institut de Sociologie de
} 
deuxième colloque, il fit un exposé sur "Présentation d'une enquête socio-démographique sur la population juive de l'agglomération de Bruxelles » et c'est lui qui, avec le professeur Usiel Oscar Schmelz, de l'Université hébraïque de Jérusalem, se chargea de l'édition des actes parus en $1972^{13}$.

D'autres lettres rendent compte de ses préoccupations scientifiques. C'est ainsi que, le 7 septembre 1994, il m'écrivait avoir passé « beaucoup de temps avec Léonty Soloveichik, Un prolétariat méconnu, Bruxelles, Paris, 1898 ; Léon Errera, Les Juifs russes, Bruxelles 1893, les socialistes belges, les antisémites comme Edmond Picard, etc.». Le 11 octobre 1995, je fus invité avec mon épouse Danièle pour ouvrir l'année universitaire avec une Conférence à deux voix sur «Les Juifs du Midi. Une histoire millénaire ", à l'occasion de la parution de notre ouvrage commun portant le même titre (paru aux édition Barthélémy d'Avignon). Ce fut une séance particulière, avec une ouverture musicale du Quatuor à cordes Arriaga, car l'Institut entamait la vingt-cinquième année d'activités (il fut inauguré le $1^{\text {er }}$ février 1972 par Emmanuel Levinas) et Willy Bok s'employa pour réaliser une très belle affiche. Deux ans plus tard, le 28 avril 1997, je présentais à l'Institut un cours sur les « Juifs de Roumanie à la veille de la Seconde Guerre mondiale », à l'occasion de la parution de mon livre Les Juifs en Roumanie (1918-1938). De l'émancipation à la marginalisation (Paris-Louvain, éditions Peeters). Quelques années plus tard, le 27 mars 2002, Willy Bok nous fit l'honneur d'animer à l'université Paul Valéry de Montpellier un «Séminaire d'Histoire religieuse et des mentalités » dans le cadre du Diplôme d'études approfondies (DEA) avec une communication intitulée « De la Haskalah au mouvement de la réforme juive en Europe (fin XVIII ${ }^{\mathrm{e}}$ début $\mathrm{XIX}^{\mathrm{e}}$ siècles)», suivie par des collègues enseignants et de nombreux étudiants.

Je souhaite insister sur la grande érudition et la générosité de notre ami, qu'il a manifestées particulièrement pendant la préparation de mon livre Les Mythes fondateurs de l'antisémitisme (paru chez Privat en

l'Université libre de Bruxelles, 1965 (368 p), pp. 89-106. Il a codirigé, avec Max Gottschalk, la publication de ce volume.

${ }^{13}$ Willy Bok, « Présentation d'une enquête socio-démographique sur la population juive de l'agglomération de Bruxelles ", dans Démographie et identité juives dans l'Europe contemporaine. Les actes du deuxième colloque sur la Vie Juive dans l'Europe contemporaine, tenu à l'Institut de Sociologie de l'Université Libre de Bruxelles, du 9 au 12 janvier 1967. Textes réunis et revus par Willy Bok et Usiel Oscar Schmelz, Éditions de l'Université de Bruxelles, 1972 (422 p.), pp. 69-72. 
2003), lorsqu'il me transmit d'utiles renseignements bibliographiques et lut une partie de mon manuscrit, me faisant bénéficier de ses observations judicieuses. Notre correspondance contient aussi beaucoup d'éléments personnels concernant aussi bien sa famille (particulièrement ses neveux Ariane et Mark-Henri Cykiert) que la mienne (Danièle et mes enfants Michaël et Sarah), et dans nos lettres, comme dans nos conversations, le "vous » s'est vite transformé en «tu », et cette amitié précieuse s'est manifestée par de très nombreux entretiens téléphoniques où la langue yiddish était souvent présente. Nos liens se sont poursuivis pratiquement jusqu'au mois d'octobre de cette année, lorsque j'ai eu la chance de le revoir à l'occasion de la conférence inaugurale « $\mathrm{Du}$ génocide des Arméniens à la Shoah. Typologie des massacres du XX siècle ». Cette conférence donnée le 19 octobre 2015 à l'Institut, avec mon collègue Gérard Dédéyan, fut introduite par le président du Conseil d'administration et l'actuel directeur, le professeur Thomas Gergely. J'ai trouvé Willy Bok amaigri, mais toujours de bonne humeur, bien qu'il se déplaçât avec une canne et avec difficulté.

En conclusion, comment résumer la personnalité de notre ami?

- En tant qu'universitaire, après des études à l'Université de Liège, à l'Université Libre de Bruxelles et à l'Université hébraïque de Jérusalem, il a été directeur du Centre d'études du judaïsme contemporain de 1'Institut de Sociologie de l'ULB (anciennement Centre National des Hautes Études Juives) et de la collection Centre National des Hautes Études Juives aux éditions de l'Université de Bruxelles (1961-1990). Professeur attaché à l'Institut d'Études du Judaïsme depuis 1972 et maitre de conférences invité à la faculté de théologie et de droit canonique de l'Université catholique de Louvain depuis 1998, il a poursuivi ses enseignements jusqu'à son décès. Il fut un enseignant d'élite - il possédait la magie des mots, la puissance du verbe, l'éloquence de la parole - toujours proche de ses étudiants.

- En tant que directeur et administrateur-fondateur de l'Institut d'Études du judaïsme (anciennement Institut universitaire d'études du judaïsme Martin Buber) de 1970 à 2002, lorsqu'il fut remplacé par le professeur Thomas Gergely, il a réussi à organiser une vaste gamme d'enseignements et, entouré d'une bonne équipe d'enseignants, mais aussi d'excellents collaborateurs administratifs, il a su transformer cet Institut, dans l'un des plus importants établissements d'enseignement d'études juives en Europe. 
Parmi les enseignants, il convient de rappeler d'abord les noms de ceux qui nous ont hélas quittés: Emmanuel Levinas, Bernhard Blumenkranz, Haïm Zafrani et Gabrielle Sed-Rajna, pour laquelle il avait une admiration et affection particulière, ainsi que les suivants qui tous devinrent ses amis: René-Samuel Sirat, Roland Goetschel, Gérard Nahon, Georges Weill, Mireille Hadas-Lebel, Daniel Tollet, Evelyne Oliel-Grausz et tant d'autres...

- En tant que chercheur, il a publié des articles importants et hormis ceux déjà cités, j'aimerais signaler ceux publiés dans l'American Jewish Year Book consacrés à la Belgique ${ }^{14}$, ceux parus dans le Dictionnaire d'histoire de la Belgique ${ }^{15}$ ou ceux parus dans les actes des Colloques du Word Union of Jewish Studies de Jérusalem ${ }^{16}$.

Auteur d'un livre sur sa communauté d'origine, Aspects de la communauté juive de Liège. Contributions à l'étude sociologique d'un milieu déterminé ${ }^{17}$, et de deux ouvrages en collaboration consacré respectivement aux Enfants nés d'un mariage dont l'un des conjoints est juif $^{18}$ et à La presse juive en Belgique et aux Pays-Bas ${ }^{19}$, il a dirigé plusieurs livres, en collaboration avec Max Gottschalk: Histoire juive contemporaine (1964, 104 p.) ; Martin Buber. L'homme et le philosophe (1968, 74 p.); Le renouveau de la culture juive (1968, 51 p.); en collaboration avec Jean-Pierre Francotte, Les Relations de la communauté israélite de Belgique avec le pouvoir central (1972, 67 p.) et en collaboration avec Maurice Krajzman, L'image des juifs et du judaïsme. Une analyse des manuels d'histoire de l'enseignement secondaire officiel en Belgique (1973, 78 p.). L'ensemble de ces titres

\footnotetext{
14 « Belgium » dans l'American Jewish Year Book 1974-1975. A Record of Events and Trends in American and World Jewish Life, New York, Philadelphia, 1974, The American Jewish Committee and the Jewish Publication Society of America, pp. 437454 ; « Belgium », dans American Jewish Year Book 1977, Ibid., 1976, pp. 388-409.

${ }^{15}$ Plusieurs articles dans Dictionnaire d'histoire de la Belgique. Vingt siècles d'histoire. Les hommes, les faits, publié sous la direction de Hervé Hasquin, Bruxelles, DidierHatier, 1988.

16 Willy Bok, «La population juive en Belgique au $\mathrm{XIX}^{\mathrm{e}}$ siècle. Aspects sociodémographiques », dans Papers in Jewish Demography,1969, edited by U.O. Schmelz, P. Glikson and S. Della Pergola, World Union of Jewish studies and Institute of Contemporary Jewry of the Hebrew University of Jerusalem, 1973, pp. 43-82.

17 Willy Bok, Aspects de la communauté juive de Liège. Contributions à l'étude sociologique d'un milieu déterminé, Liège, 1959, 146 p.

${ }^{18}$ (En collaboration avec Berty Goldberg), Dualité culturelle et appartenance. Les enfants nés d'un mariage dont l'un des conjoints est juif. Analyse comparative dans un milieu universitaire, Bruxelles, 1970, $81 \mathrm{p}$.

${ }^{19}$ (En collaboration avec Maurice Krajman), La Presse juive en Belgique et aux PaysBas. Histoire et analyse de contenu, Bruxelles, 1975, 209 p.
} 
atteste l'intérêt de leur auteur pour les aspects sociologiques, démographiques, historiques et culturels du judaïsme.

Dans le domaine des publications, étant donné sa vaste érudition, l'on peut regretter qu'il n'a pas pu achever et faire paraître plusieurs synthèses consacrées au judaïsme européen et américain qu'il connaissait fort bien. En d'autres termes, nous regrettons qu'il n'ait pas donné toute sa mesure. Ceci peut s'expliquer par son investissement exceptionnel dans la vie de l'Institut où il était présent tout le temps. Tous les enseignants peuvent témoigner - et moi en premier - qu'il leur réservait un accueil d'une rare qualité, les accompagnant jusqu'à leur hôtel et prenant souvent des repas avec eux.

- En tant qu'homme, il fut d'abord et surtout un homme de cœur, porteur d'une grande expérience et d'une générosité infinie. Modeste, il ne voyait jamais de mal dans l'autre, le prochain ou le lointain et, souvent dans nos discussions, il se prononça pour un dialogue ouvert, nécessaire et exigeant aussi bien avec les chrétiens qu'avec les musulmans. Toutes les grandes causes, surtout la fraternité et la paix entre les peuples, particulièrement la paix nécessaire entre les Israéliens et les Palestiniens, lui tenaient particulièrement à cœur.

Dans la conclusion de son article sur Chaïm Perelman publié en 1985, Willy Bok écrivait :

Si l'Institut Universitaire d'Études du Judaïsme Martin Buber a pu remplir la mission qu'il s'est assignée de contribuer par l'étude au développement de la connaissance du monde juif, il le doit beaucoup à la sollicitude jamais démentie de son président du Conseil scientifique. Chaïm Perelman n'a pas cessé de prodiguer ses encouragements, en payant de sa personne. Sa présence aux nombreuses réunions de l'Institut, ses avis éclairés et sa bonté foncière manqueront cruellement. L'Institut honorera sa mémoire en continuant l'œuvre à laquelle il s'est consacré avec tant de dévouement. ${ }^{20}$

Nous pouvons et devons reprendre ces mêmes jugements de valeur et les attribuer à Willy Bok.

Toujours affable, prêt à offrir aussitôt son amitié surtout aux jeunes, il a su transmettre par son enseignement les valeurs essentielles $\mathrm{du}$ judaïsme et de l'éthique juive. Un homme qui vous accueillait toujours le sourire aux lèvres, un homme plein de noblesse d'âme, un mensch dans la tradition du yidishkeit de l'Europe de l'Est, tel fut Willy Bok que j'ai connu.

${ }^{20}$ « Chaïm Perelman ou la double fidélité », op. cit. p. 76. 


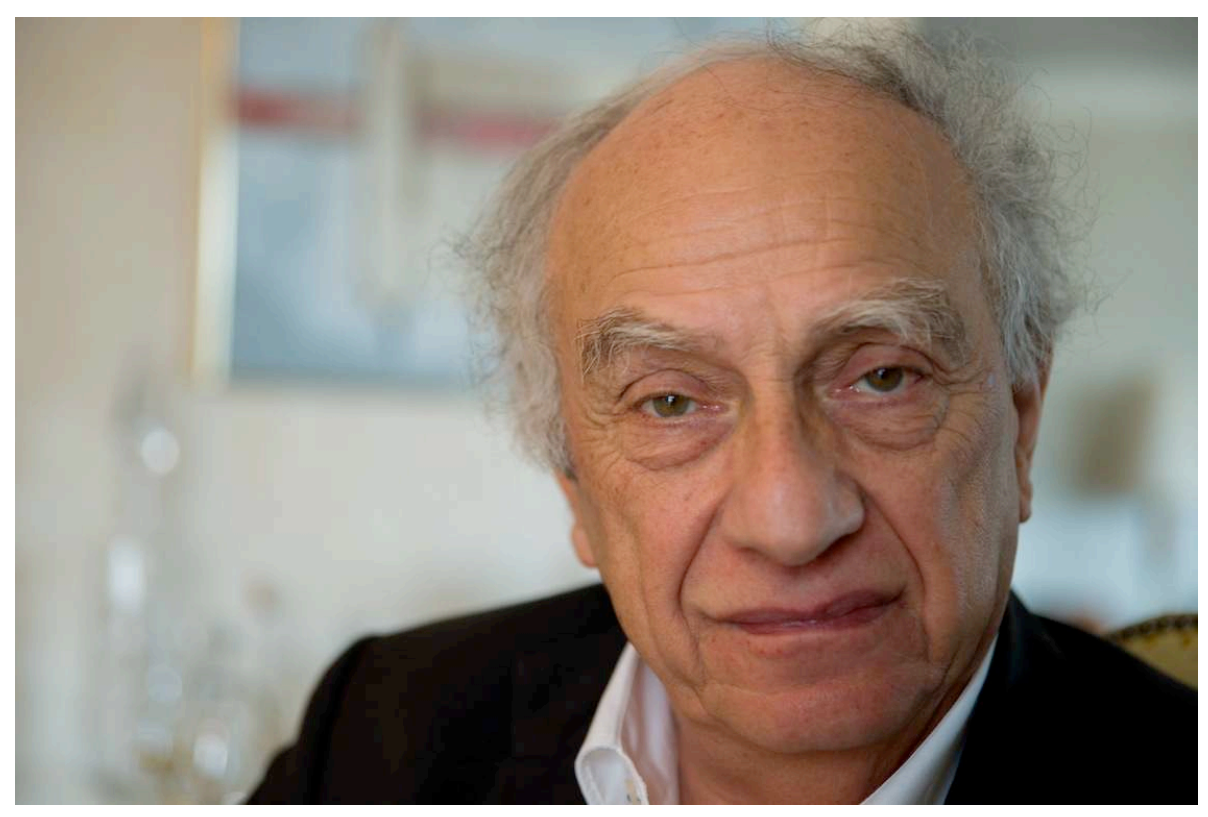

Willy Bok, Bruxelles, 2015

(Photo Max Cykiert $($ ) M. Cykiert)

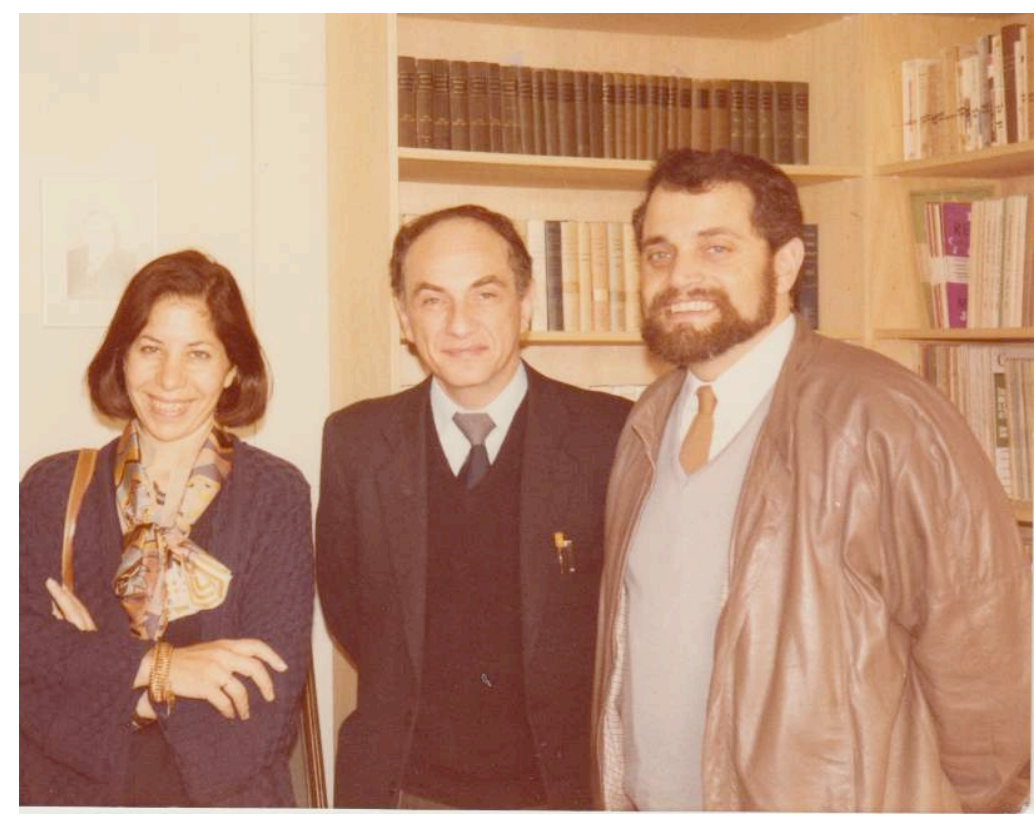

Willy Bok entouré de Danièle et Carol Iancu, Bruxelles, Institut Martin Buber, 1985

(C) Carol Iancu) 